\title{
Evaluación preliminar de un sistema de recirculación de aguas para un prototipo implementado en la producción de tilapia roja (Oreochromis sp.)
}

\author{
Preliminary evaluation of a water recirculation system for a \\ prototype implemented in the production of red tilapia (Oreochromis \\ sp.) \\ Ricardo Valenzuela ${ }^{1 *}$, Paula Martínez ${ }^{2}, J_{o h n}$ Jairo Arevalo ${ }^{3}$
}

\begin{abstract}
Resumen
El objetivo de este estudio fue analizar y evaluar parámetros de la calidad del agua como potencial de hidrogeno $(\mathrm{pH})$, temperatura $\left({ }^{\circ} \mathrm{c}\right)$, oxígeno disuelto $(\mathrm{ppm})$, turbidez $(\mathrm{NTU})$, alcalinidad $\left(\mathrm{mgCaCO}_{3} / \mathrm{L}\right)$, nitratos $\left(\mathrm{mgNO}_{2} / \mathrm{L}\right)$, amonio total (ppm), Demanda Bioquímica de Oxigeno $\left(\mathrm{mgO}_{2} / \mathrm{L}\right)$ y Demanda Química de Oxigeno $\left(\mathrm{mgO}_{2} / \mathrm{L}\right)$ en un sistema cerrado de recirculación implementado para la producción de tilapia roja (Oreochromis sp.) durante 90 días. El sistema está compuesto de un tanque plástico de 500 litros, dos filtros de arena, 20 biobolas, dos bombas de $60 \mathrm{~W}$, un soporte de 1.5 metros de altura y un tanque plástico de 60 galones en donde están 10 plantas macrófitas (buchón de agua). Los peces fueron alimentados durante ese periodo de tiempo tres veces al día con el $2 \%$ de su peso promedio. Los parámetros de $\mathrm{pH}$ y amonio se registraron a diario con la ayuda de indicadores, el oxígeno disuelto con oxímetro y los demás fueron enviados al laboratorio. Se utilizó dos métodos de filtración, mecánico y biológico de los cuales se obtuvo porcentajes de remoción de solidos muy altos, manteniendo un ambiente de oxigenación óptimo para el adecuado desarrollo de los peces.

Palabras clave: calidad del agua; nitrificación; cultivo de tilapia; filtro de arena; biofloc.
\end{abstract}

\section{Abstract}

The objective of this study was to analyze and evaluate parameters of water quality such as hydrogen potential $(\mathrm{pH})$, temperature $\left({ }^{\circ} \mathrm{C}\right)$, dissolved oxygen $(\mathrm{ppm})$, turbidity $(\mathrm{NTU})$, alkalinity $(\mathrm{mgCaCO} 3 / \mathrm{L})$, nitrates $(\mathrm{mgNO} 2) /$ L), ammonium (ppm), Biochemical Oxygen Demand (mgO2 / L) and Chemical Oxygen Demand (mgO2 / L) in a closed recirculation system implemented for the production of red tilapia (Oreochromis sp.) For 90 days. The system is composed of a plastic tank of 500 liters, two sand filters, 20 biobolas, two pumps of 60W, a support of 1.5 meters high and a plastic tank of 60 gallons where there are 10 macrophyte plants (buchón de Water). The fish were fed during that period of time three times a day with $2 \%$ of their average weight. The $\mathrm{pH}$ and ammonium parameters were recorded daily with the help of indicators, dissolved oxygen with oximeter and the others were sent to the laboratory. Two mechanical and biological filtration methods were used, from which very high removal percentages of solids were obtained, maintaining an optimal oxygenation environment for the proper development of the fish.

Keywords: water quality; nitrification; tilapia culture; sand filter; biofloc.

\footnotetext{
${ }^{1}$ Estudiante de Ingenieria AgrícolaUniversidad Surcolombiana. Av. Pastrana Borrero carrera 1a Neiva. E-mail: richar913@hotmail.com ${ }^{2}$ MSc. Profesor Dpto ingeniería ambiental Corhuila. Calle 8 No. $32-49$ Neiva. E-mail: paula.martinez@ corhuila.edu.co

${ }^{3}$ MSc. Profesor DptoIngeniero Agrícola Universidad Surcolombiana. Av. Pastrana Borrero carrera 1a Neiva. E-mail:john.arevalo@usco.edu.co** (Autor de correspondencia)
} 


\section{Introducción}

Los sistemas cerrados de recirculación de aguas implementados para la acuicultura ha sido una alternativa de producción sostenible que ha tenido bastante aceptación, pero su limitante siempre está en los métodos de filtración para eliminación de los desechos producto de esta actividad y el consumo energético (Timmons, 2007). Estos sistemas se componen de 5 principales etapas entre procesos y operaciones que permiten sostener la calidad del agua, las cuales son circulación de agua, remoción de sólidos, biofiltración, oxigenación y eliminación de gases (Gallego, 2010).

En esta investigación se utilizó la tilapia roja (Oreochromis sp.) por su capacidad para adaptarse a diferentes cambios de calidad de agua considerando unos rangos bastante amplios de tolerancia como lo describe Saavedra (2006) en la tabla 1 y además por ser una de las tres especies más cultivadas a nivel nacional e internacional (DANE,2014).

Tabla 1. Parámetros de calidad de agua para tilapia roja (Oreochromis sp.)

\begin{tabular}{|c|c|c|}
\hline PARÁMETROS & UNIDADES & $\begin{array}{c}\text { RAN } \\
\text { GOS }\end{array}$ \\
\hline Temperatura & ${ }^{\circ} \mathrm{C}$ & $25-32$ \\
\hline Oxígeno disuelto & $\mathrm{mg} / \mathrm{L}$ & $5-9$ \\
\hline $\mathrm{pH}$ & Unidades de $\mathrm{pH}$ & $6-9$ \\
\hline Alcalinidad Total & $\mathrm{mg} / \mathrm{L}$ & $50-150$ \\
\hline Dureza Total & $\mathrm{mg} / \mathrm{L}$ & $80-110$ \\
\hline Calcio & $\mathrm{mg} / \mathrm{L}$ & $60-120$ \\
\hline Nitritos & $\mathrm{mg} / \mathrm{L}$ & $0-0.1$ \\
\hline Nitratos & $\mathrm{mg} / \mathrm{L}$ & $1.5-2$ \\
\hline Amonio Total & $\mathrm{mg} / \mathrm{L}$ & $0-0.1$ \\
\hline Hierro & $\mathrm{mg} / \mathrm{L}$ & $0.05-$ \\
\hline Fosfatos & $\mathrm{mg} / \mathrm{L}$ & 0.2 \\
\hline $\begin{array}{c}\text { Dióxido de } \\
\text { carbono }\end{array}$ & $\mathrm{mg} / \mathrm{L}$ & 0.2 \\
\hline $\begin{array}{c}\text { Sulfuro de } \\
\text { Hidrogeno }\end{array}$ & $\mathrm{mg} / \mathrm{L}$ & $0-0.01$ \\
\hline
\end{tabular}

Fuente: Saavedra Martínez, M. A. (2006). Manejo del cultivo de tilapia. Managua, Nicaragua.

Los aspectos que tienen mayor importancia son: $\mathrm{pH}$, temperatura, alcalinidad total, Turbidez, Nitratos, Nitritos, Amonios, Oxígeno disuelto, demanda biológica de oxígeno y demanda química de oxígeno (Colt, 2006).
La tilapia crece mejor en aguas de $\mathrm{pH}$ neutro o levemente alcalino. Su crecimiento se reduce en aguas ácidas y toleran hasta un $\mathrm{pH}$ de 5 . Los niveles bajos de $\mathrm{pH}$ producen mortalidad en un periodo de 3 a 5 horas, por fallas respiratorias y cutáneas (Brito \& Rodríguez, 2009).

La temperatura afecta directamente la tasa metabólica de los peces en especial cuando existen oscilaciones diarias de por encima de $5^{\circ} \mathrm{C}$, cuando la una aumenta la otra también lo hace, y por consiguiente el consumo de oxígeno. (Castillo 1994; Chimits, 1998; Fiztsimmons, 2000; como se citó en Borja et al.,2006). Para la tilapia el rango óptimo de temperatura es de $28^{\circ}-30^{\circ}$ Celsius $\left({ }^{\circ} \mathrm{C}\right)$; cuando la temperatura disminuye de $\operatorname{los} 15^{\circ} \mathrm{C}$, la especie deja de comer hasta morir como lo observo Valbuena \& Cruz, (2006) que peces de $25 \mathrm{~g}$ a $18{ }^{\circ} \mathrm{C}$ consumieron $176 \pm 15 \mathrm{mg} \cdot \mathrm{kg}^{-1} \cdot \mathrm{h}^{-1}$, en tanto que a $30^{\circ} \mathrm{C}$ el consumo promedio fue de $508 \pm 120 \mathrm{mg} \cdot \mathrm{kg}^{-1} \cdot \mathrm{h}^{-1}$. El efecto de la temperatura sobre el consumo de $\mathrm{O} 2$ mostró una relación directamente proporcional; es decir, a mayor temperatura del agua se presentó mayor consumo de O2. Otro rol importante de este parámetro como lo afirma Dawson \& Murphy, (1972) es la influencia que tiene en la velocidad de remoción de la nitrificación como lo hace en todas las cinéticas de reacciones químicas y biológicas.

La turbidez debe mantenerse con valores inferiores a 25 NTU aunque según Boyd (1998), el agua puede estar de 10 hasta 40 NTU.

Los nitritos son un producto intermedio de la transformación del amoníaco en nitrato por la actividad bacteriana. El nitrito es tóxico en los peces, mermando la capacidad de la hemoglobina para transportar oxígeno, por lo cual su toxicidad está relacionada con el oxígeno disuelto en agua (Carvajal 2014), un incremento en el $\mathrm{CO}_{2}$ puede disminuir el $\mathrm{pH}$ a un valor por debajo de 6.5 , lo que puede llevar a toxicidad de nitrito a través de la formación de ácido nitroso $\left(\mathrm{HNO}_{2}\right)$.

El Amoníaco en el agua existe en dos formas, como iones de Amoníaco $\left(\mathrm{NH}_{4}+\right)$, los cuales no son tóxicos, y como Amoníaco tóxico no ionizado $\left(\mathrm{NH}_{3}\right)$. La proporción relativa de uno o el otro depende de la temperatura del agua y el $\mathrm{pH}$. La toxicidad del amonio aumenta al incrementar el $\mathrm{pH}$, debido a que en aguas básicas la posibilidad de excretar el amoniaco se ve limitada, por la escasez de 
concentración de protones $(\mathrm{H}+)$, por lo tanto, se produce una intoxicación por esta sustancia, caso contrario ocurre en agua acidas, donde hay mayor prevalencia de amonio ionizado $\left(\mathrm{NH}_{4}+\right)$, citado por Carvajal , (2014).

La Tilapia tiene una tolerancia a bajas concentraciones de oxígeno disuelto, teniendo una presión parcialmente baja es capaz de saturarse de Oxígeno y más aún, de reducir su consumo si la concentración es inferior a $3 \mathrm{mg} / \mathrm{l}$ usando un metabolismo semi-anaerobio, soportando niveles de 1 $\mathrm{mg} / \mathrm{l}$ e incluso menor por períodos cortos, (Morales como se citó en Martínez. et al, 2015) aunque lo recomendable es de 5-6 mg / L para evitar el estrés de los peces (Bernstein, 2011, Rakocy, 2007, citado por Sumeth et al., 2017). Actualmente el monitoreo de este parámetro es tan rigoroso que se están creando sensores conectados a aplicaciones móviles para tener más control ante cualquier variación que ocurra (Dussan et al., 2016).

La demanda biológica de oxígeno (DBO) es la cantidad de oxígeno que los microorganismos, especialmente bacterias (aerobias o anaerobias facultativas: Pseudomonas, Escherichia, Aerobacter, Bacillus), hongos y plancton, consumen durante la degradación de las sustancias orgánicas contenidas en el agua para Boyd (1998), debe tener valores de $5 \mathrm{mgO}_{2} / \mathrm{L}$ a $20 \mathrm{mgO}_{2} / \mathrm{L}$.

La demanda química de oxígeno (DQO) es la cantidad de oxígeno necesario para oxidar la materia orgánica por medios químicos y convertirla en dióxido de carbono y agua. El valor de la DQO siempre será inferior al de la DBO debido a que muchas sustancias orgánicas pueden oxidarse químicamente pero no biológicamente. Los valores aceptables están por entre $40 \mathrm{mgO}_{2} / \mathrm{l}-80 \mathrm{mgO}_{2} / \mathrm{l}$ (Boyd, 1998).

\section{Materiales y metodos}

Para la realización de este proyecto se contó con el apoyo financiero y tecnológico del tecno parque seccional Neiva- Huila ubicada en la diagonal 20 №38-16, barrio Buganviles bajo la dirección del SENA.

En el sistema se sembraron 20 alevinos de tilapia roja (Oreochromis sp.) con una longitud aproximada de $3,5 \mathrm{~cm}$ y de un peso promedio de 9 gramos, los cuales fueron adquiridos en la finca la esperanza, vereda el bajo juncal municipio de CampoalegreHuila a los 60 días después de haber eclosionado. El tiempo de la evaluación fue de 90 días. El agua que se utilizó para el llenado del tanque de los peces es proveniente del acueducto de la ciudad de NeivaHuila pero que se dejó decantar naturalmente durante 8 días antes de ingresar a los peces, con ese método se garantiza que el cloro se evapore.

La región donde se desarrolló el prototipo se encuentra en una zona de vida de bosque seco tropical, en donde los índices de evaporación están alrededor de $4 \mathrm{~mm} /$ día por lo tanto se debe hacer recargas del $2 \%$, recomendado por García, citado en Gallego (2010).

Para el óptimo desarrollo de este proyecto de la siguiente manera:

Recolección de datos: La temperatura se midió con un termómetro, oxígeno disuelto con un oxímetro y el amonio junto con el pH se midió con los indicadores, estos parámetros se tomaron diariamente en el tanque de producción $\left(\mathrm{P}_{1}\right)$ y en el filtro con plantas $\left(\mathrm{P}_{3}\right)$ como se puede ver en la figura 1 .

Muestreo: para las pruebas de laboratorio se tomó dos muestras de agua por cada punto de inspección.

P1: salida del tanque de producción

P2: salida del filtro de arena

P3: salida del filtro biológico

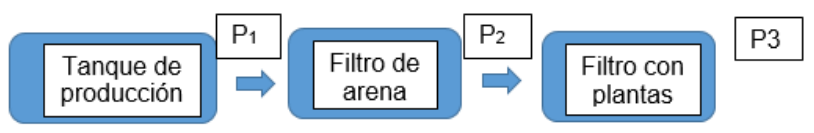

Figura 1. Esquema de puntos de muestreo del prototipo

Pruebas de laboratorio: los parámetros de $\mathrm{DBO}_{5}$, DQO, $\mathrm{pH}$, turbiedad, alcalinidad total y nitritos se realizaron en el laboratorio DIAGNOSTICAMOS, ubicados en la carrera 11 No. 07-45 Neiva-Huila, a los 90 días de haber iniciado el proceso. 


\section{Resultados y discusión}

Los datos de la tabla 1,2 y 3 son los resultados de las pruebas de laboratorio tomadas a los 90 días de haber iniciado el proyecto y son los primeros en analizar seguidos de las figuras 1,2 y 3 que corresponden al seguimiento diario del amonio (ppm), oxígeno disuelto $(\mathrm{mg} / \mathrm{L})$, temperatura $\left({ }^{\circ} \mathrm{c}\right) \mathrm{y}$
$\mathrm{pH}$ en el tanque de los peces y filtro con plantas para tener un control del proceso general del tratamiento del agua.

En la tabla 1, se presentan los resultados de laboratorio de la muestra extraída del tanque de los peces

Tabla 1. Resultados de laboratorio de la muestra de agua proveniente del tanque de peces

\begin{tabular}{|c|c|c|c|}
\hline PARAMETRO & UNIDADES & RESULTADO & RANGO OPTIMO \\
\hline Alcalinidad total & $\mathrm{mg} \mathrm{CaCO} 3 / \mathrm{L}$ & 26,88 & $50-150$ \\
\hline $\begin{array}{c}\text { Demanda biológica } \\
\text { de oxigeno }\end{array}$ & $\mathrm{mg} \mathrm{O} / \mathrm{L}$ & $<10$ & $0-30$ \\
\hline $\begin{array}{c}\text { Demanda química } \\
\text { de oxigeno }\end{array}$ & $\mathrm{mg} \mathrm{O} / \mathrm{L}$ & 45 & $0-40$ \\
\hline Nitritos & $\mathrm{mg} \mathrm{NO} 2 / \mathrm{L}$ & 0,466 & 0,01 \\
\hline $\mathrm{pH}$ & Unidades de $\mathrm{pH}$ & 5,88 & $6-9$ \\
\hline Turbiedad & $\mathrm{NTU}$ & 1,92 & $0-5$ \\
\hline
\end{tabular}

Fuente: laboratorio de aguas, Diagnosticamos S.A. Neiva-Huila (2017)

De acuerdo con los resultados, se afirma que los peces están en un ambiente ligeramente acido por su nivel de pH y alcalinidad. Según Nicovita (2017) estos son indicadores que el agua no se encuentra en buenas condiciones, el $\mathrm{pH}$ de 5.75 y la alcalinidad de $20.43 \mathrm{mg} \mathrm{CaCO}_{3} / \mathrm{L}$ pueden afectar el aparato respiratorio y la pigmentación de los peces. La demanda química de oxigeno muestra que el agua presenta valores de agua contaminada con capacidad para auto durarse y la demanda biológica de oxigeno cataloga el agua como aceptable (Boyd, 1973). El nitrito es el valor más alarmante porque pasa los limites óptimos, pero su toxicidad no es elevada por tener un $\mathrm{pH}$ ácido y la oxigenación alta. Con respecto a la turbiedad, las partículas suspendidas en el agua también absorberán calor adicional de la luz solar lo cual ocasionará que el agua sea más caliente. El agua caliente no es capaz de guardar tanto oxígeno como el agua fría, así que los niveles de Oxígeno Disuelto bajaron, especialmente cerca de la superficie. Los niveles superiores a 5 NTU, no son aptos por la dificultad que tienen los peces para ver el alimento por lo tanto este se desperdicia y va al fondo del tanque, favoreciendo más la contaminación. En las biobolas se acumulan microalgas, ciliados, rotíferos, nematodos y bacterias de los géneros Sphingomonas, Pseudomonas, Bacillus, Nitrospira, Nitrobacter y la levadura Rhodotorula sp.que sirven como fuente de alimento y que además contribuyen al mejoramiento de la calidad del agua del cultivo. (Monroy et al., 2013)

Según Mariluz (2015) obtuvo en su investigación, oxígeno disuelto entre $7.54 \pm 0.21$ a $7.73 \pm 0.31$ $\mathrm{mgO}_{2} / \mathrm{L}, \mathrm{pH}$ entre $7.23 \pm 0.34$ a $7.58 \pm 0.35$, nitritos entre $0.36 \pm 0.03$ a $0.39 \pm 0.042 \mathrm{mg} / \mathrm{L}$ manteniendo una temperatura promedio de $24^{\circ} \mathrm{c}$ durante un periodo de 180 días. Para Gallego (2010) obtuvo niveles de alcalinidad de $54.46 \pm 0.57 \mathrm{mg} / \mathrm{L}$, oxígeno disuelto de $4.86 \pm 0.07 \mathrm{mgO}_{2} / \mathrm{L}, \mathrm{pH}$ de $6.95 \pm 0.07$, demanda química de oxigeno de $51 \pm 58 \mathrm{mg} / \mathrm{L}$, nitritos de $0.250 \pm 0.006 \mathrm{mg} / \mathrm{L}$ y solidos suspendidos de $3.64 \pm 0.03 \mathrm{mg} / \mathrm{L}$ manteniendo una temperatura promedio de $16.76 \pm 0.13{ }^{\circ} \mathrm{C}$ en un cultivo de trucha Arco Iris durante 150 días. Ingle de la Mora et al. (2003), en un estudio con Tilapia del Nilo (Oreochromis niloticus) y de trucha arco iris (Oncorhynchus mykiss) en sistema cerrado de recirculación de agua mantuvo los peces con un $\mathrm{pH}$ 8.4 \pm 0.13 ; demanda química de oxigeno $34.3 \pm 47.4$ $\mathrm{mg} / \mathrm{L}$; temperatura $18.9 \pm 1.2{ }^{\circ} \mathrm{c}$; oxígeno disuelto $6.4 \pm 0.5 \mathrm{mg} / \mathrm{L}$ y nitrógeno amoniacal total $0.32 \pm 0.22$ $\mathrm{mg} / \mathrm{L}$ en un periodo de 120 días.

En la tabla 2, se encuentran los resultados de los parámetros de laboratorio de la muestra proveniente. 
del filtro de arena.

Tabla 2. Resultados de laboratorio de la muestra de agua proveniente del filtro de arena

\begin{tabular}{|c|c|c|c|}
\hline PARAMETRO & UNIDADES & RESULTADO & RANGO OPTIMO \\
\hline Alcalinidad total & $\mathrm{mg} \mathrm{CaCO3/L}$ & 22,58 & $50-150$ \\
\hline $\begin{array}{c}\text { Demanda biológica } \\
\text { de oxigeno }\end{array}$ & $\mathrm{mg} \mathrm{O} 2 / \mathrm{L}$ & $<10$ & $0-30$ \\
\hline $\begin{array}{c}\text { Demanda química } \\
\text { de oxigeno }\end{array}$ & $\mathrm{mg} \mathrm{O} / \mathrm{L}$ & $<10$ & $0-40$ \\
\hline Nitritos & $\mathrm{mg} \mathrm{NO} / \mathrm{L}$ & 0,528 & 0,01 \\
\hline $\mathrm{pH}$ & Unidades de $\mathrm{pH}$ & 6,15 & $6-9$ \\
\hline Turbiedad & NTU & 1,26 & $0-5$ \\
\hline
\end{tabular}

Fuente: laboratorio de aguas. Diagnosticamos S.A. Neiva-Huila (2017)

El pH y la alcalinidad muestran que el agua, aunque sigue siendo acida, al pasar por los filtros de arena, subió ligeramente sus niveles debido a la composición del material filtrante. La demanda biológica y química de oxigeno siguen catalogando el agua como contaminada, pero con capacidad de auto depurarse. El nitrito no está en el rango de valores permisibles pero su toxicidad no es letal por los altos niveles de oxigenación. La turbiedad nos demuestra que el agua se encuentra con mayor transparencia.

La utilización de arena como material filtrante se ha usado para tratamiento de aguas para consumo humano como lo hizo Cargua (2014) donde al pasar el agua contaminada por el filtro redujo el $\mathrm{pH}$ de 7.54 a 7.27 ; turbiedad de 1.96 a 0.70 NTU; alcalinidad de 119 a $40 \mathrm{mg} / \mathrm{L}$ y nitritos mantiene su nivel de 0.01 $\mathrm{mg} / \mathrm{L}$; también Valdiviezo (2013) obtuvo una reducción de $\mathrm{pH}$ de 6.75 a 6.59 ; la alcalinidad de 293.67 a $260 \mathrm{mg} / \mathrm{L}$; la turbiedad y los nitritos se mantiene igual, $0.22 \mathrm{NTU}$ y $0.01 \mathrm{mg} / \mathrm{L}$. Con respecto a la piscicultura, Gallego, (2010) obtuvo una reducción de $\mathrm{pH}$ de $7.13 \pm 0.07$ a $6.99 \pm 0.07$, alcalinidad de $51.42 \pm 0.56$ a $46.11 \pm 0.56 \mathrm{mg} / \mathrm{L}$, nitritos de $0.312 \pm 0.006$ a $0.201 \pm 0.006 \mathrm{mg} / \mathrm{L}$, demanda química de oxigeno de $46.6 \pm 0.44$ a $43.81 \pm 0.37 \quad \mathrm{mgO}_{2} / \mathrm{L}$ y solidos suspendidos de $3.73 \pm 0.04$ a $2.06 \pm 0.033 \mathrm{mg} / \mathrm{L}$. Ingle de la Mora et al.(2003) en su trabajo encontró que el pH se mantuvo en un nivel de 8.4 ; mientras que el nitrógeno amoniacal total disminuyo de 0.32 a $0.04 \mathrm{mg} / \mathrm{L}$ y la demanda química de oxigeno aumento de 34.3 a $37.7 \mathrm{mg} / \mathrm{L}$ durante un periodo de 120 días. Para Tsukuda et al.(2015), consiguió una eficiencia de remoción de nitratos de $26.9 \pm 0.9 \%$, eliminando $402 \pm 14 \mathrm{~g} \mathrm{NO}_{3} \mathrm{~N} / \mathrm{m}^{3}$ aunque las concentraciones de nitrito- $\mathrm{N}$ y nitrógeno total de amonio aumentaron levemente 11 y $13 \%$ porque no hubo el suficiente carbono orgánico para la degradación de estos.

En la tabla 3, se presentan los resultados de laboratorio de la muestra proveniente del filtro con las plantas acuáticas.

Tabla 3. Resultados de las pruebas de la muestra de agua proveniente del filtro con plantas

\begin{tabular}{|c|c|c|c|}
\hline PARAMETRO & METODO & UNIDADES & RESULTADO \\
\hline $\begin{array}{c}\text { Alcalinidad total } \\
\text { Demanda biológica de } \\
\text { oxigeno }\end{array}$ & $\begin{array}{c}\text { SM 52320B } \\
\text { método C } \\
\text { oxigeno }\end{array}$ & $\mathrm{mM} \mathrm{CaCO} / \mathrm{L}$ & 22.58 \\
\hline $\begin{array}{c}\text { Demanda química de } \\
\text { Nitritos }\end{array}$ & $\mathrm{mg} 220 \mathrm{D}$ & $\mathrm{mg} \mathrm{O}_{2} / \mathrm{L}$ & $<10$ \\
\hline PH & SM 4500-HOH & $\mathrm{mg} \mathrm{NO}_{2} / \mathrm{L}$ & 0.528 \\
\hline Turbiedad & SM 2130B & Unidades de PH & 6.15 \\
\hline
\end{tabular}

Fuente: laboratorio de aguas. Diagnosticamos S.A. Neiva-Huila (2017) 
El agua sube el nivel de $\mathrm{pH}$ por el aporte de la arena de los filtros a un nivel cada vez más cercano a la neutralidad. Según Zou et al. (2016), el pH con mayor desnitrificacion se obtiene cuando esta en 6.0 porque un ambiente acido inhibe agentes distrificantes. La demanda biológica y química de oxigeno se encuentran en un rango aceptable, sin rastro de contaminación, de acuerdo a Boyd \& Hillary (1997) por las concentraciones de clorofila del fitoplancton,plantas acuticas y algas. El nitrito aumentó un poco debido a que las raíces de las plantas pudieron desprender este compuesto al ser movidas en algunas ocasiones por cuestiones de mantenimiento. El agua se encuentra en un estado de transparencia con una turbiedad de $1,26 \mathrm{NTU}$ propicia para el desarrollo de esta planta (Thi Nguyen et al., 2015).

Según Gomez \& Pinzon (2012), el buchón de agua (Eichornia crassipes) es un fitorremediador natural que disminuye los nutrientes que conducen a la eutroficacion del agua como lo indica en su trabajo donde el $\mathrm{pH}$ se acidifico hasta llegar a 3, el oxigeno disuelto en $6.6 \mathrm{mg} / \mathrm{L}$ y nitritos en $0.01 \mathrm{mg} / \mathrm{L}$. El agua contaminada al ser tratada con este sistema tienda a neutralizar su pH y remover hasta en $89 \%$ fenoles, $71 \%$ fosforo y $87 \%$ nitratos (Muñoz , 2016).

En la investigacion de Rubim et al. (2015), comparo el porcetaje de cobertura de esta planta y su relacion con la calidad del agua observo que los parámetros evaluados se redujeron después del paso del biofiltro bajo la presencia de E. crassipes: $73.7 \%$ para conductividad eléctrica, $15 \%$ para $\mathrm{pH}, 84.5 \%$ para turbidez, $86.8 \%$ para nitrito, $69 \%$ para fósforo total y $77.8 \%$ para ortofosfato.

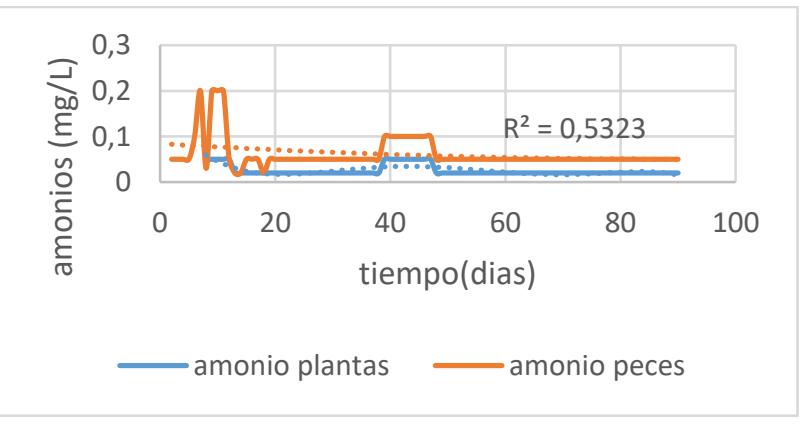

Figura 1. Concentración de amonio diaria durante el experimento
En la figura 1 se puede observar el comportamiento del amonio durante todo el experimento. En los primeros 15 días, los niveles de amonio fueron elevados en el tanque de los peces debido a que el sistema aún no se había madurado.

La maduración consiste en dejar desarrollar bacterias nitrificadoras en las biobolas para que oxiden los nutrientes disueltos en el agua y después ser asimilados por los peces, de acuerdo a Gallego (2010) esto se da cuando la concentración amoniacal total se vuelve constante por debajo de $0.2 \mathrm{mg} \mathrm{N}$ $\mathrm{NH}_{3} / \mathrm{L}$.

En ese tiempo hubo una mortalidad del $10 \%$. Teniendo en cuenta la figura 1, el nivel promedio de amonio en el tanque de los peces es de $0.06 \pm 0.03$ $\mathrm{mg} / \mathrm{L}$ y para las plantas es de $0.02 \pm 0.01 \mathrm{mg} / \mathrm{L}$ mostrando diferencia estadística significativa entre los dos procesos (filtro de arena y plantas). El porcentaje de remoción de amonio es de $66.67 \%$.

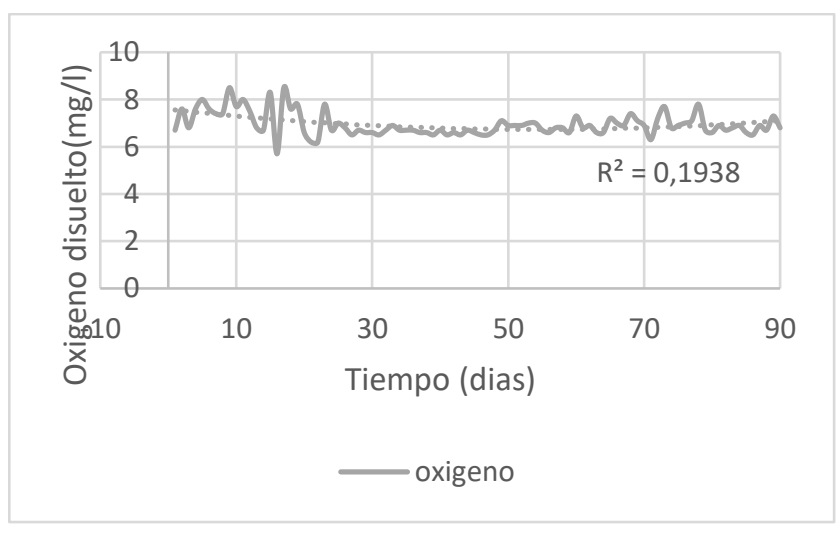

Figura 2. Concentración de oxígeno disuelto diaria durante el experimento.

La figura 2 muestra la concentración de oxígeno disuelto en el tiempo con un promedio de $6.95 \pm 0.5$ $\mathrm{mg} / \mathrm{l}$. Por ser el indicador más importante, fue tomado todos los días y en la jornada de la mañana. El resultado demuestra que el agua esta con el oxígeno adecuado con base a lo dicho por Galo P., afirmando que debe ser mayor de $4.5 \mathrm{mg} / \mathrm{L}$ (citado por Brito S., 2009 p.5) y para Kubitza mayor a $3.5 \mathrm{mg} / \mathrm{L}$ (citado por Carvajal E, 2014 p.17).

La temperatura promedio del agua de los peces es de $26.2 \pm 1.5^{\circ} \mathrm{c}$ como se puede observar en la figura 3 , manteniéndose en los rangos admisibles que según 
Kubitza debe estar entre 28 y $32^{\circ} \mathrm{C}$ (citado por Carvajal 2014 p.17) y para Saavedra (2006) entre 25 y $32^{\circ} \mathrm{c}$. Aunque en la ciudad de Neiva-Huila la temperatura promedio oscila entre 32 y $34^{\circ} \mathrm{c}$, el agua no alcanzo a tener esos valores.

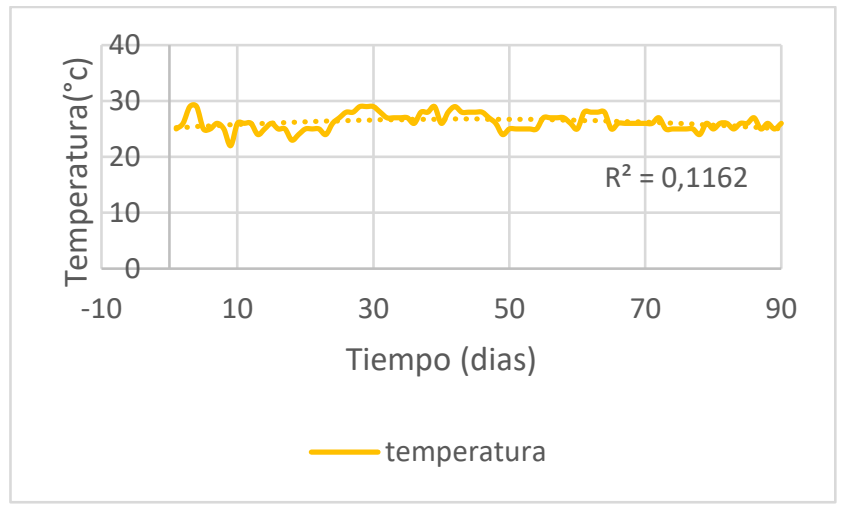

Figura 3. Valores de temperatura diaria durante el experimento

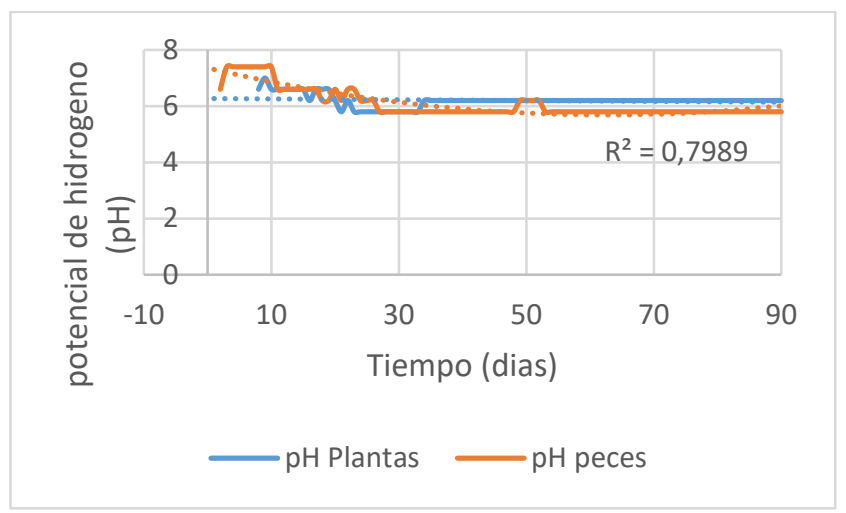

Figura 4. Potencial de hidrogeno diaria durante el experimento.

Como se observa en la figura 4 , el $\mathrm{pH}$ del agua de los peces es en promedio de $6.08 \pm 0.49$ y el $\mathrm{pH}$ para el tratamiento con las plantas es de 6.2 \pm 0.22 , arrojando diferencias estadísticamente significativas

Estos niveles se encuentran en los rangos permisibles de acuerdo a Nicovita, Kubitza y Southern Regional Aquaculture Center, en donde establecen que un $\mathrm{pH}$ entre 6.0 hasta 8.5 son los óptimos para el desarrollo de los peces (citado por Carvajal, 2014).

\section{Conclusiones}

Con base en los resultados obtenidos en el desarrollo del sistema de recirculación implementado en la producción de tilapia roja propuesto en este trabajo, se demuestra que esta tecnología es una alternativa viable para ahorro del recurso hídrico en la producción de alimento de alta calidad y gran valor nutritivo. La configuración del tren de tratamiento utilizado (filtro de arena y filtro con plantas) se considera aceptable para producir 20 de tilapias roja a talla comercial ( $250 \mathrm{~g}$ ) lo que significó un ahorro de agua de hasta el $98 \%$, comparado con la tecnología vigente. El prototipo permite el $0.5 \%$ del consumo de agua de un sistema abierto (tecnología de cultivo tradicional).

La maduración del sistema se alcanzó cuando el nivel del nitrógeno amoniacal total se mantuvo en valores menores a $0.2 \mathrm{mg} / \mathrm{L}$; porque aumento la producción de fitoplancton y algunas bacterias benéficas que regulaban este nutriente en el agua.

La eficiencia global de remoción obtenida del sistema fue de $71.49 \%$ para turbiedad, $66.67 \%$ para amonio y $25.7 \%$ para nitritos. El filtro de arena disminuyo los niveles de demanda química de oxígeno, nitrato y turbiedad por lo contrario pasa con el filtro con plantas en donde la alcalinidad y la turbiedad disminuyo y el $\mathrm{pH}$ junto con los nitritos aumentaron levemente. Sin embargo, la eficiencia lograda permite contar con agua tratada de calidad suficiente para el cultivo de Tilapia roja.

Esta investigación permite estimar la producción de contaminantes que se generarán durante el ciclo de producción a partir de la biomasa cultivada. Con base en los resultados es posible calcular la producción de amonio en función de la biomasa de tilapia a cultivar, lo que permite realizar un diseño recirculación de aguas más seguro y apegado a la realidad.

\section{Agradecimientos}

Se agradece la colaboración del Tecnoparque nodo Neiva del Servicio Nacional de Aprendizaje SENA por el apoyo financiero y brindarnos sus instalaciones para el desarrollo de este trabajo. 


\section{Referencias Bibliográficas}

Borja Gallardo, F. J., Gonzalez Salcedo, L. O., \& Quintero de Vallejo, V. E. (2006). Evaluación de alternativas para climatización de estanques con energía solar para cultivo de tilapia roja (oreochromis sp), localizados en la zona fría del valle del cauca, colombia. Revista Facultad Nacional de Agronomía Medellín, 59(1). Articulo. Obtenido de http://www.redalyc.org/articulo.oa?id=17991407401 4

Boyd, C. E. \& Hillary S. E. (1997). Dynamics of Pond Aquaculture. Pond Dynamics / Aquaculture Collaborative Research Support Programme (CRSP), 166-167. libro. Obtenido de https://books.google.com.co/books?id=m_EczUbFnD MC\&pg=PA166\&dq=chemical+and+biological+dem and+of+water+oxygen+in+aquiculture+production\& $\mathrm{hl}=\mathrm{es} \& \mathrm{sa}=\mathrm{X} \& \mathrm{ved}=0 \mathrm{ahUKEwj}-$

2uq7wqDgAhXFq1kKHV66Dk0Q6AEIKjAA\#v=on epage\&q=chemical\%20and\%20biological\%20deman $\mathrm{d} \% 20$ of $\% 20$ water\%20 oxygen $\% 20 \mathrm{in} \% 20$ aquiculture $\% 20$ production $\& \mathrm{f}=$ false

Boyd, C. E. (1998). Water quality for pond aquaculture. Aurbn : International Center for aquaculture and Aquatic Environment Alabama Aqucultural Experiment station. Libro. Obtenido de http://www.ag.auburn.edu/fish/documents/Internation al_Pubs/R\&D\%20Series/43\%20-

$\%$ 20Water\%20Quality\%20for\%20Pond\%20Aquacult ure.pdf consultado el 16 mayo 2018

Brito Suárez, F. G. \& Rodríguez L. C. (2009). Efecto de la reutilización del agua en la crianza y producción de tilapia roja: Universidad del Azuay. Tesis . Obtenido de http://dspace.uazuay.edu.ec/handle/datos/584

Cargua Lopez, M. (2014). Diseño de una planta de potabilizacion de agua en la comuna Ainche del Canton Chambo. Riobamba: Escuela Politecnica de Chimborazo. Tesis. Obtenido de http://dspace.espoch.edu.ec/handle/123456789/3418

Carvajal Echeverri, J. P. (2014). Comparacion de parametros zootecnicos y de calidad de agua de tres sistemas de precria de tilapia roja (Oreochromis Spp.) en el Municipio de Puerto Triunfo. Caldas-
Antioquia: Corporacion Universitaria Lasallista.Tesis. Obtenido de http://hdl.handle.net/10567/1511

Colt, J. (2006). Water quality requirements for reuse systems. Aquacultural Engineering, 34, 143156. Articulo. Obtenido de doi:https://doi.org/10.1016/j.aquaeng.2005.08.011

DANE. (2014). El cultivo de la tilapia roja (Orechromis sp.) en estanques de tierra,fuente de proteína animal de excelente calidad. Informe. Obtenido de https://www.dane.gov.co/files/investigaciones/agrope cuario/sipsa/insumos_factores_de_produccion_mar_2 014.pdf consultado 2 de junio 2018.

Dawson, R., \& Murphy, K. (1972). The temperature dependency of biological denitrification. Water Research, 71-83. Articulo. Obtenido de doi:https://doi.org/10.1016/0043-1354(72)90174-1

Dussán, S. A., Vanegas, O. O., Chavarro, A. F., Molina, J. J. (2016). Diseño e implementacion de un prototipo electronico para monitoreo de parametros fisico-quimicos en cultivo de tilapia a traves de un aplicacion movil. Informador Técnico, 80(1), 49-60. doi:https://doi.org/10.23850/22565035.322.

Gallego Alarcon, I. (2010). Evaluacion y modelacion de un tren de tratamiento de agua residual acuicola con recirculacion y del cultivo de trucha Arco Iris alimentado por cosecha pluvial. Toluca-Mexico: Universidad Autonoma del estado de Mexico. Tesis doctoral. Obtenido de doi: 10.13140 / rg.2.1.3720.1761

Gomez Sierra, H., \& Pinzon castañeda , G. P. (2012). Analisis de la mitigacion del impacto ambiental en el lago del parque la Florida, por fitorremediacion usando Buchon de agua. Bogota D.C: Universidad Militar Nueva Granada. Tesis. Obtenido de http://hdl.handle.net/10654/7129

Ingle de la Mora, G., Villarreal Delgado, E., Arredondo Figueroa, J. L., Ponce Palafox, J. T., \& Barriga Sosa, I. (2003). Evaluación de algunos parámetros de calidad del agua en un sistema cerrado de recirculación para la acuicultura, sometido a diferentes cargas de biomasa de peces. Hidrobiologica, 13(4), 247-253. Articulo. Obtenido de http://www.redalyc.org/articulo.oa?id=57813402 
Rubim M.A.L., P.R. Isolino Sampaio \& P Parolin (2015). Biofilter efficiency of Eichhornia crassipes in wastewater treatment of fish farming in Amazonia. Phyton, International Journal of Experimental Botany, 84(1), 244-251. Obtenido de http://ppct.caicyt.gov.ar/index.php/phyton/article/vie w/8133/7342 consultado el 2 de junio 2018 consultado el 2 junio 2018

Mariluz Fernandez, A. A. (2015). Evaluacion de los parametros productivos y de calidad de agua en el cultivo de tilapia Oreochromis niloticus en sistemas de recirculacion cerrada en laboratorio. Callao: Universidad Nacional del Callao. Tesis . Obtenido de http://repositorio.unac.edu.pe/handle/UNAC/1000

Martinez Suarez, J. L., Perez Delgado, A., Sanchez Armas, S. E., \& Diaz Orejan, E. T. (2015). Propuesta de un medidor de oxigeno disuelto en cultivos de Tilapia. Puebla- Mexico: Universidad Tecnologica de Tehuacan. Articulo. Obtenido de http://studylib.es/doc/7799815/propuesta-de-unmedidor-de-oxigeno-disuelto-en-cultivos-d. consultado el 3 junio 2018

Monroy Dosta, M., De Lara Andrade, R., Castro Mejía, J., Castro Mejía, G., \& Coelho Emerenciano, M. G. (2013). Composición y abundancia de comunidades microbianas asociadas al biofloc en un cultivo de tilapia. Revista de Biología Marina y Oceanografía, 48(3), 511-520. doi:DOI 10.4067/S0718-19572013000300009

Muñoz Cruz, F. A. (2016). Evaluacion de la remocion de fenoles, fosforo y nitratos en agua residual domestica rural en Brusela Huila, a partir de un sistema de fitorremediacion con buchones de agua(eichhornia crassipes). Pitalito-Huilla: UNAD. Tesis. Obtenido de http://hdl.handle.net/10596/8581

Nicovita. (18 de 08 de 2017). Industria acuicola. Obtenido Manual de crianza de tilapia: http://www.industriaacuicola.com/biblioteca/Tilapia/ Manual\%20de\%20crianza\%20de\%20tilapia.pdf consultado el 2 junio 2018

Valbuena-Villarreal R.D \& Cruz-Casallas P.E. (2006). Efecto del peso corporal y temperatura del agua sobre el consumo de oxígeno de tilapia roja (Oreochromis sp). ORINOQUIA, 57-63. Articulo.
Obtenido

de

http://www.redalyc.org/pdf/896/89610107.pdf

Valdiviezo Sanchez, P. S. (2014). Diseño de un sistema de tratamiento para la potabilizacion del agua en la parroquia San Andres del Canton Guano, Provincia de Chimborazo. Riobamba: Escuela de ingenieria de Chimborazo. Tesis. Obtenido de http://dspace.espoch.edu.ec/handle/123456789/3220

Saavedra Martinez , M. A. (2006). Manejo del cultivo de tilapia. Managua, Nicaragua. Libro. Obtenido de http://www.crc.uri.edu/download/MANEJO-DEL-

CULTIVO-DE-TILAPIA-CIDEA.pdf consultado el 2 junio 2018

Sumeth Wongkiew, Zhen $\mathrm{Hu}$, Kartik Chandran, Jae Woo Lee \& Samir Kumar Khanal (2017). Nitrogen transformations in aquaponic systems: A review. Aquacultural Engineering, 76, 9-19. Articulo. Obtenido doi:https://doi.org/10.1016/j.aquaeng.2017.01.004

Thi Nguyen Tien Hanh; Boets Pieter;Lock Koen; Damanik Ambarita Minar Naomi ;Eurie ForioMarie Ane ;Sasha Peace ;Dominguez-Granda Luis Elvin ;Thi Hoang Thu Huong ;Everaert Gert \& Goethals Peter LM (2015). Habitat suitability of the invasive water hyacinth and its relation to water quality and macroinvertebrate diversity in a tropical reservoir. Limnologica - Ecology and Management of Inland Waters, 52, 67-74. doi:https://doi.org/10.1016/j.limno.2015.03.006

Timmons, M. E. (2007). Recirculating aquaculture. Cayuga Aqua Ventures. Libro. Obtenido de https://doi.org/10.1002/9781118250105.ch11

Tsukuda, S., Christianson, L., Kolb, A., Saito, K., \& Summerfelt, S. (2015). Heterotrophic denitrification of aquaculture effluent using fluidized sand biofilters. Aquacultural Engineering, 64, 49-59. doi:https://doi.org/10.1016/j.aquaeng.2014.10.010

Zou, Y., Hu, Z., Zhang, J., Xie, H., Christophe, G., \& Fang, Y. (Junio de 2016). Effects of pH on nitrogen transformations in media-based aquaponics. Bioresource Technology, 210, 81-87. doi:doi.org/10.1016/j.biortech.2015.12.079 